\title{
Estudo das propriedades reológicas de nanofluidos à base de etilenoglicol e óxido de grafeno
}

\section{Study of rheological properties of nanofluids based on ethylene glycol and graphene oxide}

\author{
Marielle Mara da Silva ${ }^{1}$, Bruno Rocha Santos Lemos ${ }^{2}$, \\ Marcelo Machado Viana ${ }^{3}$
}

\footnotetext{
${ }^{1}$ Pontifícia Universidade Católica de Minas Gerais (PUC Minas), Av. Dom José Gaspar, 500, Campus Coração Eucarístico - Departamento de Engenharia Química - Instituto Politécnico (IPUC), CEP: 30535-901, Belo Horizonte, MG, Brasil.

${ }^{2}$ Pontifícia Universidade Católica de Minas Gerais (PUC Minas), Av. Dom José Gaspar, 500, Campus Coração Eucarístico - Departamento de Física e Química - Instituto de Ciências Exatas e Informática (ICEI), CEP: 30535-901, Belo Horizonte, MG, Brasil.

${ }^{3}$ Universidade Federal de Minas Gerais (UFMG), Av. Antônio Carlos, 6627, Campus da Pampulha - Departamento de Química - Instituto de Ciências Exatas (ICEx), CEP: 31270-901, Belo Horizonte, MG, Brasil. e-mail: marielle.mara@outlook.com, brunorslemos@gmail.com, marcelomachado@ufmg.br
}

\section{RESUMO}

O óxido de grafeno (GO) é um nanomaterial de carbono que apresenta propriedades únicas e dentre elas, a excelente condutividade térmica que pode ser explorada em nanocompósitos, como também em nanofluidos. O etilenoglicol (ETG) é um fluido de troca térmica que pode ter suas propriedades térmicas potencializadas pela adição de GO. Porém, tanto o nível de dispersão de GO quanto a reologia da suspensão, podem influenciar as características dos nanofluidos, como o fluxo, a estabilidade e a transferência térmica. Assim, o estudo reológico de nanofluidos ETG/GO é importante para o entendimento da estabilidade coloidal dessas suspensões e do potencial de aplicação como fluido de transferência térmica. Neste trabalho, GO sintetizado pelo método de Hummers modificado foi utilizado para preparar nanofluidos ETG/GO para estudos reológicos. Técnicas instrumentais como microscopia eletrônica de varredura (MEV), difração de raios X (DRX), espectroscopia na região do infravermelho por transformada de Fourier (FTIR), microscopia eletrônica de transmissão (MET) e termogravimetria (TGA), confirmaram a obtenção de GO com cerca de 4 folhas de grafeno e funcionalização de $38 \%$ com grupos oxigenados. A superfície hidrofílica do GO permitiu que as nanofolhas tivessem boa compatibilidade com o ETG. Foram investigados os perfis de curvas de fluxo das suspensões de nanofluidos ETG/GO, e estes foram classificados como: não Newtonianos e pseudoplásticos pelo modelo de Ostwald-de Waele. A inserção de GO ao ETG, aumentou a viscosidade e o módulo de armazenamento (G') em $21 \%$ e $8 \%$, respectivamente. As propriedades aprimoradas evidenciam o potencial de uso de nanofluidos de GO para aplicações em sistemas térmicos.

Palavras-chave: Reologia; Óxido de grafeno; Etilenoglicol; Nanofluidos.

\begin{abstract}
Graphene oxide (GO) is a carbon nanomaterial that has unique properties, including excellent thermal conductivity, which can be explored in nanocomposites, as well as in nanofluids. Ethylene glycol (ETG) is a heat transfer fluid that can have its thermal properties enhanced by the addition of GO. However, the GO dispersion capacity and suspension rheology can influence the characteristics of nanofluids, such as flow, stability and heat transfer. The rheological study of ETG/GO nanofluids is important for understanding the colloidal stability of these suspensions and the potential for application as heat transfer fluids. In this work, GO synthesized by modified Hummers method was used to prepare ETG/GO nanofluids for rheological studies. Instrumental techniques such as scanning electron microscopy (SEM), X-ray diffraction (XRD), Fourier transform infrared spectroscopy (FTIR), transmission electron microscopy (TEM) and thermogravimetry (TGA),
\end{abstract}


confirmed the GO synthesis with approximately 4 layers of graphene nanosheets and $38 \%$ of functionalization with oxygenated groups. The hydrophilic surface of GO nanosheets allowed good compatibility with ETG. The flow curve profiles of the ETG/GO nanofluid suspensions were investigated and classified as: nonNewtonian and pseudoplastic by the Ostwald-de Waele model. The insertion of GO to the ETG, increased the viscosity and storage module (G') in $21 \%$ and $8 \%$, respectively. The improved properties show potential of using GO nanofluids for applications in thermal systems.

Keywords: Rheology; Graphene oxide; Ethylene glycol; Nanofluids.

\section{INTRODUÇÃO}

Fluidos convencionais utilizados na indústria, como água e etilenoglicol (ETG), desempenham papéis essenciais em sistemas térmicos, porém, estes possuem características térmicas intrinsecamente ruins devido à baixa condutividade térmica [1], fator essencial para extensas aplicações de transferência de calor. Com o desenvolvimento tecnológico e a intensificação de processos industriais, a deficiência de sistemas de troca térmica eficientes se tornou uma demanda tecnológica contemporânea, tendo o viés tanto na otimização dos equipamentos quanto na capacidade térmica dos fluidos de trabalho e/ou fluidos de troca térmica [2].

Foram realizados estudos sobre a adição de partículas sólidas aos fluidos bases, a princípio em microescala e observou-se que os compostos gerados contribuíam na melhoria do desempenho das propriedades térmicas, porém geravam problemas como entupimentos de capilares, aumento do atrito e perda de carga. Posteriormente, os esforços se concentraram na adição de partículas nanométricas, formando nanofluidos [24]. O nanofluido é um fluido preparado pela dispersão de partículas sólidas de tamanho nanométrico no fluido de transferência de calor tradicional, como água ou ETG, para aumentar a condutividade térmica, a viscosidade, a transferência de calor por convecção e redução ao atrito. Os nanofluidos podem ainda, aumentar o desempenho nas trocas térmicas, reduzindo os custos de manutenção e aumentando a sua viabilidade de aplicação [5-6]. Os nanofluidos têm sido utilizados em várias aplicações de transferência de calor, como em coletores solares, automóveis, armazenamento de energia, trocadores e dissipadores de calor, e geradores de energia [7-9]. Foi relatado [3] que a condutividade térmica do nanofluido é superior comparada ao fluido base e depende do tamanho, forma, fração volumétrica, composição e propriedades térmicas das nanopartículas presentes.

O grafeno e seus derivados têm atraído diversas atividades de pesquisa, pois este material possui propriedades mecânicas, ópticas e térmicas, que são favoráveis para o desenvolvimento de sistemas altamente estáveis, que mantenham suas propriedades por longos períodos e que superem os limites de desempenho dos sistemas já utilizados [10]. Já o óxido de grafeno (GO), tem sua estrutura baseada no grafeno, porém modificada com grupos funcionais oxigenados, como epóxidos, hidroxilas, carboxilas e outros [11,12]. Segundo THAKUR et al.[13] e GUDARZI [14], a inserção desses grupos funcionais oxigenados, promove mudanças na hibridação de alguns átomos de carbono, gerando folhas de GO altamente estáveis em suspensão aquosa. Essa capacidade do GO para formar sistemas coloidais estáveis é devida a sua estrutura atômica bidimensional e à repulsão eletrostática entre as folhas, efeito da elevada densidade de cargas negativas, devido à ionização das funções oxigenadas inseridas no processo de oxidação química [14]. O GO possui notáveis propriedades como: elevada resistência mecânica e condutividade térmica, dispersão em água e solventes orgânicos, entre outras [11, 12]. Assim, a preparação de suspensões estáveis contendo GO é uma etapa fundamental da engenharia no uso desses fluidos convencionais de transferência de calor com características aprimoradas [5], como por exemplo, o aumento da condutividade térmica e melhor controle sobre os fenômenos de corrosão e erosão [15], fornecendo ganhos em propriedades reológicas.

A reologia como ciência do fluxo e da deformação da matéria, descreve a inter-relação entre força, deformação, temperatura e tempo [16], fornecendo informações sobre o comportamento e estabilidade de um fluido, sendo que este pode estar no estado líquido, sólido ou gasoso [17]. Apesar do amplo uso da reologia para caracterizações mecânicas, os estudos reológicos também servem como uma ferramenta poderosa para determinar a relação entre a microestrutura local e as propriedades macroscópicas de um determinado material [16].

Os fluidos são caracterizados de acordo com duas classes: Newtonianos - aqueles que possuem uma relação linear entre tensão de cisalhamento e a taxa de cisalhamento, ou seja, a viscosidade apenas depende da temperatura e pressão; e os não Newtonianos - aqueles os quais o comportamento não é linear nas condições impostas, sendo os mais comuns os fluidos pseudoplásticos e dilatantes, em que os valores de viscosidade diminuem e aumentam conforme a taxa de cisalhamento aumenta, respectivamente [16-18]. A caracterização reológica é geralmente realizada por meio de medições em fluxo de cisalhamento linear oscilatório, fluxo de cisalhamento constante e fluxo de cisalhamento transiente. A partir dessas medições, informações impor- 
tantes sobre a conformação local das folhas de GO em dispersão podem ser avaliadas [19-23].

Modelos reológicos são utilizados para caracterizar os fluidos de acordo com o comportamento reológico, e usualmente utilizam expressões matemáticas que correlacionam a tensão de cisalhamento e a taxa de deformação. O modelo de Newton fornece uma equação que descreve o fluxo ideal, sendo a tensão de cisalhamento e a taxa de deformação proporcionalmente constante, relação característica de fluidos Newtonianos. Modelos de Bingham, Ostwald-de Waele, Herschell-Buckley, Arrhenius, entre outros; fornecem equações que descrevem os fluidos não Newtonianos, como por exemplo: suspensões coloidais, solução de polímeros, pasta, gel, e outros [18]. Deste modo, os modelos reológicos permitem simular a resposta do fluido e predizer o comportamento reológico em relação à composição e às condições de processo.

O nível de dispersão das nanopartículas pode influenciar nas propriedades reológicas, devido às características superficiais das nanopartículas. NACIFY et al. [5] encontraram propriedades viscoelásticas únicas para dispersões de GO mais concentradas, sendo classificadas como uma nova classe de materiais, sendo assim, torna-se evidente a necessidade de investigações no sentido de avaliar o comportamento reológico destes nanofluidos, visando a otimização de sistemas e a potencialização da aplicabilidade desta tecnologia.

Contudo, a reologia é uma ferramenta essencial para o estudo do comportamento dos nanofluidos, e mesmo que seja extensa a literatura sobre a reologia de materiais baseados em nanotubos de carbono, tanto na forma de dispersão quanto em compósito, ainda é pouco relatado sobre a reologia das dispersões de óxido de grafeno e seus efeitos sobre o fluido base no qual se aplica este material [19, 23, 24].

No presente estudo, investigamos as propriedades reológicas de nanofluidos de ETG/GO formulados com diferentes concentrações de GO, este possuindo aproximadamente 4 folhas de grafeno e com um grau de funcionalização de $38 \%$, propriedades que favorecem a dispersão a longo prazo do GO em ETG [25].

\section{MATERIAIS E MÉTODOS}

\subsection{Materiais}

Grafite expansível foi adquirido da Asbury Carbon. Ácido fosfórico $\left(\mathrm{H}_{3} \mathrm{PO}_{4}\right)$, ácido sulfúrico $\left(\mathrm{H}_{2} \mathrm{SO}_{4}\right)$, permanganato de potássio $\left(\mathrm{KMnO}_{4}\right)$, peróxido de hidrogênio $\left(\mathrm{H}_{2} \mathrm{O}_{2}\right)$ e ácido clorídrico $(\mathrm{HCl})$ foram adquiridos da Sigma-Aldrich. Etilenoglicol P.A. foi comprado da empresa Synth.

\subsection{Síntese do óxido de grafeno (GO)}

Os procedimentos foram embasados nos autores MARCANO et al. [11], utilizando o método de Hummers modificado. Inicialmente, o grafite expansível da empresa Asbury, foi expandido termicamente durante 30 minutos a $800^{\circ} \mathrm{C}$. Para a obtenção de GO, grafite expandido (EG) foi adicionado a uma solução contendo $\mathrm{H}_{2} \mathrm{SO}_{4}$ e $\mathrm{H}_{3} \mathrm{PO}_{4}$ na proporção volumétrica de 9:1. Posteriormente, a mistura foi deixada sob agitação magnética a $100^{\circ} \mathrm{C}$ durante 2 horas. Em seguida, adicionou-se $\mathrm{KMnO}_{4}$ na proporção mássica de 1:5 (grafite: $\mathrm{KMnO}_{4}$ ); e essa mistura foi deixada sob agitação magnética por 2 horas a $50^{\circ} \mathrm{C}$. Posteriormente, adicionou-se $50 \mathrm{~mL}$ de água destilada e a mistura foi submetida a um banho de ultrassom à uma potência de $250 \mathrm{~W}$, por 2 horas. Acrescentou-se $20 \mathrm{~mL}$ de $\mathrm{H}_{2} \mathrm{O}_{2} 30 \%$ v/v. e após a retirada do sobrenadante, foi adicionado um volume de $\mathrm{HCl} 10 \%$ v/v igual ao de água destilada da etapa anterior. Em seguida, a solução foi lavada com água destilada até neutralização do meio. A suspensão aquosa de óxido de grafeno obtida foi liofilizada para a obtenção do GO sólido.

\subsection{Preparo de nanofluidos de ETG/GO}

Segundo ZHANG et al. [21] e SHU et al. [22], em faixas de concentrações muito baixas, as folhas de GO dispersas possuem fracas interações entre si, porém, se o GO excede uma concentração crítica, as dispersões de GO podem se transformar em um cristal líquido nemático, exibindo outro comportamento reológico. Desta forma, para analisar uma ampla faixa de concentrações, foram preparadas 5 amostras com as seguintes concentrações de GO: $0 \mathrm{mg} \cdot \mathrm{mL}^{-1} ; 0,5 \mathrm{mg} \cdot \mathrm{mL}^{-1} ; 2,5 \mathrm{mg} \cdot \mathrm{mL}^{-1} ; 4,5 \mathrm{mg} \cdot \mathrm{mL}^{-1}$ e $8 \mathrm{mg} \cdot \mathrm{mL}^{-1}$. As amostras de óxido de grafeno em etilenoglicol foram sonicadas durante 1 hora à uma potência de $250 \mathrm{~W}$, para garantir uma boa dispersão [10]. Devido aos grupos funcionais aderidos na estrutura do GO, não foi necessário uso de aditivos ou surfactantes, pois, a superfície hidrofílica permitiu que as nanofolhas de GO tivessem boa compatibilidade com o ETG.

As identificações dos nanofluidos e suas respectivas concentrações, estão apresentadas na Tabela 1 abaixo. 
Tabela 1: Concentrações das amostras de nanofluidos ETG/GO.

\begin{tabular}{c|c}
\hline CÓDIGO & CONCENTRAÇÃO $\left(\mathbf{m g} \cdot \mathbf{m L}^{-1}\right)$ \\
\hline ETG & 0,0 \\
\hline ETG/GO-1 & 0,5 \\
\hline ETG/GO-2 & 2,5 \\
\hline ETG/GO-3 & 4,5 \\
\hline ETG/GO-4 & 8,0 \\
\hline
\end{tabular}

\subsection{Caracterização estrutural e morfológica do GO}

A técnica de microscopia eletrônica de varredura (MEV) foi utilizada para obter imagens da morfologia e estrutura das amostras de grafite e GO em um equipamento JEOL (modelo JSM IT 300). Na técnica de difração de raios X (DRX), amostras de grafite e GO foram analisadas utilizando-se o equipamento Shimadzu, modelo XRD7000 X-Ray Diffractometer, com radiação $\mathrm{CuK}_{\alpha}, \lambda=0,15406 \mathrm{~nm}$. As amostras foram analisadas por espectroscopia vibracional na região de Infravermelho com Transformada de Fourier (FTIR) utilizandose o equipamento Perkin Elmer (FTIR RXI) com acessório ATR de diamante, na faixa de 400 a $4000 \mathrm{~cm}^{-1}$. As imagens de microscopia eletrônica de transmissão (MET) foram obtidas em um equipamento Tecnai G220 - FEI SuperTwin $200 \mathrm{kV}$. As análises termogravimétricas (TGA) foram feitas no equipamento o DSC60Plus Shimadzu, DTG-60H, utilizando atmosfera inerte, taxa de aquecimento de $10^{\circ} \mathrm{C} \cdot \mathrm{min}^{-1}$ e um fluxo de $50 \mathrm{~mL} \cdot \mathrm{min}^{-1}$.

\subsection{Caracterização reológica dos nanofluidos de GO}

As análises reológicas foram executadas no equipamento Anton Paar Modular Compact Rheometer 502(MCR 502). Nos testes realizados foi utilizado a geometria de placas paralelas de diâmetro $50 \mathrm{~mm}$ e True Gap de $1,000 \mathrm{~mm}$, a temperatura constante de $25^{\circ} \mathrm{C}$. Foram realizados quatro ensaios: (i) curva de viscosidade, (ii) varreduras dinâmicas de deformação a uma frequência constante de $1 \mathrm{rads}^{-1}$, (iii) varreduras dinâmicas de deformação a uma amplitude constante e (iv) varreduras dinâmicas de deformação em função da temperatura.

\section{RESULTADOS E DISCUSSÕES}

\subsection{Estudo morfológico}

As imagens obtidas por MEV Figura 1(a,b) mostram o grafite e grafite expandido (EG), respectivamente. A Figura 1(a) representa a morfologia do grafite antes do processo de expansão, onde se observa os "flakes" com comprimento médio de $823 \mu \mathrm{m}$. A Figura 1(b) mostra o grafite após expansão, sendo nítida a mudança de morfologia, uma vez que, a expansão proporciona o aumento do espaçamento entre as folhas de grafeno, devido a saída dos agentes intercalantes que quando vaporizam, aumentam o seu volume promovendo essa expansão. O grafite expandido tem baixa densidade e uma morfologia tipo "worm" devido à sua forma longa e torácica, como mostrado na Figura 1(b).

Após a oxidação e esfoliação, observa-se a morfologia das folhas de GO produzidas aa Figura 1(c). É possível observar a espessura reduzida das folhas formadas, que apresentam certo grau de dobramento. De acordo com LI et al. [26], isto ocorre devido ao processo de oxidação, no qual induz a mudança de hibridização $\mathrm{sp}^{2}$ para $\mathrm{sp}^{3}$ dos átomos de carbono, assim o plano bidimensional da folha de GO pode ser distorcido por átomos de carbono que estão ligados à grupos hidroxilas presentes no plano basal. Após a secagem do nanofluido, nota-se que não há evidência de aglomeração das folhas de GO (Figura 1(d)), o que contribui para a estabilidade coloidal da dispersão formada [21, 27]. 


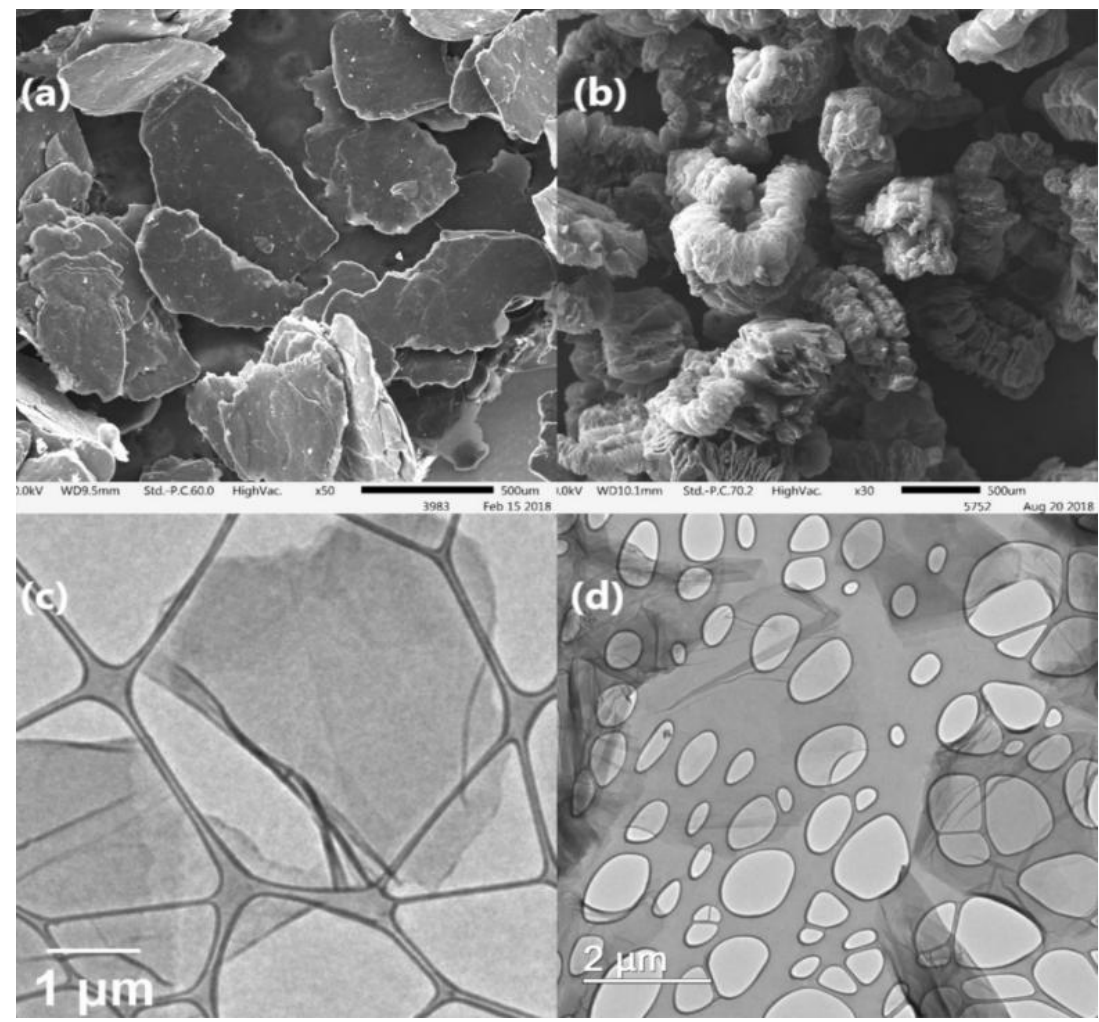

Figura 1: Micrografias de MEV para (a) grafite e (b) grafite expandido. Imagens de MET para (c) GO como preparado e (d)GO após secagem do nanofluido.

\subsection{Estudo estrutural}

Na Figura 2(a), temos o difratograma de raios X para a amostra de grafite e GO, no qual é possível observar o aumento significativo do espaçamento entre as folhas de grafeno, uma vez que, o grafite sofre uma expansão térmica e posteriormente o grafite expandido é oxidado. O GO exibe uma distância maior entre as folhas de grafeno, sendo esta variação da distância atribuída à presença de grupos funcionais na folha de grafeno [26, 27]. O grafite mostra um pico de difração nítido e intenso a $2 \theta=26,5^{\circ}$ correspondente ao plano (002) com espaçamento de $0,34 \mathrm{~nm}$. Porém, no caso de GO, o pico (002) se desloca para $2 \theta=10,7^{\circ}$ com aumento do espaçamento entre as folhas para $0,83 \mathrm{~nm}$, o que é atribuído à existência de funcionalidades de oxigênio nas camadas de GO devido à oxidação substancial sofrida [28]. No entanto, o difratograma do GO ainda contém o pico a $2 \theta=26,45^{\circ}$, porém com uma menor intensidade. Isto indica que a estrutura não foi totalmente expandida e oxidada, tendo domínios grafíticos presentes, pois, caso contrário haveria somente o pico em $2 \theta=10,67^{\circ}$. Assim, para o preparo dos nanofluidos somente as porções contendo GO como sobrenadante foram utilizadas. Através da DRX também foi possível determinar o número de folhas médias do GO, utilizando a equação de Scherrer [29]. Segundo MARASCHIN et al. [30], o número de folhas de grafeno presente no grafite e GO, pode ser calculado utilizando a razão da espessura do material pelo espaçamento entre as folhas de grafeno. Assim, o grafite utilizado na síntese de GO tem aproximadamente 123 folhas de grafeno e o GO obtido através do método de Hummers modificado, apresenta aproximadamente 4 folhas de grafeno.

Na curva TGA mostrada na Figura 2(b), foi observada uma perda de massa em duas etapas para o grafite. A perda de massa que se inicia abaixo de $100{ }^{\circ} \mathrm{C}$ é atribuída à remoção de moléculas de água adsorvidas na superfície dos materiais. A perda de massa para o grafite que ocorre em $802{ }^{\circ} \mathrm{C}$ é atribuída à degradação de sua estrutura. No caso da amostra de GO foram observadas quatro etapas na curva termogravimétrica. A perda de massa para o $\mathrm{GO}$ se inicia a $216^{\circ} \mathrm{C}$ e ocorre devido à liberação de grupos funcionais na superfície das folhas de grafeno, como os grupos hidroxila, carbonila e epóxi; a decomposição dessas funcionalidades de oxigênio é responsável pela liberação de $\mathrm{CO}$ e $\mathrm{CO}_{2}$. Esse evento térmico mostra que o grau de funcionalização do material é de 38\%. Houve perda de massa em $624{ }^{\circ} \mathrm{C}$ e foi atribuída à degradação da estrutura carbônica residual, com perda de $35 \%$ da massa total. A $802{ }^{\circ} \mathrm{C}$ ocorre uma perda de massa de $6 \%$, o que está relacionado à degradação da estrutura grafítica, ainda presente. Este resultado é corroborado pela técnica de DRX (Figura 2(a)). 


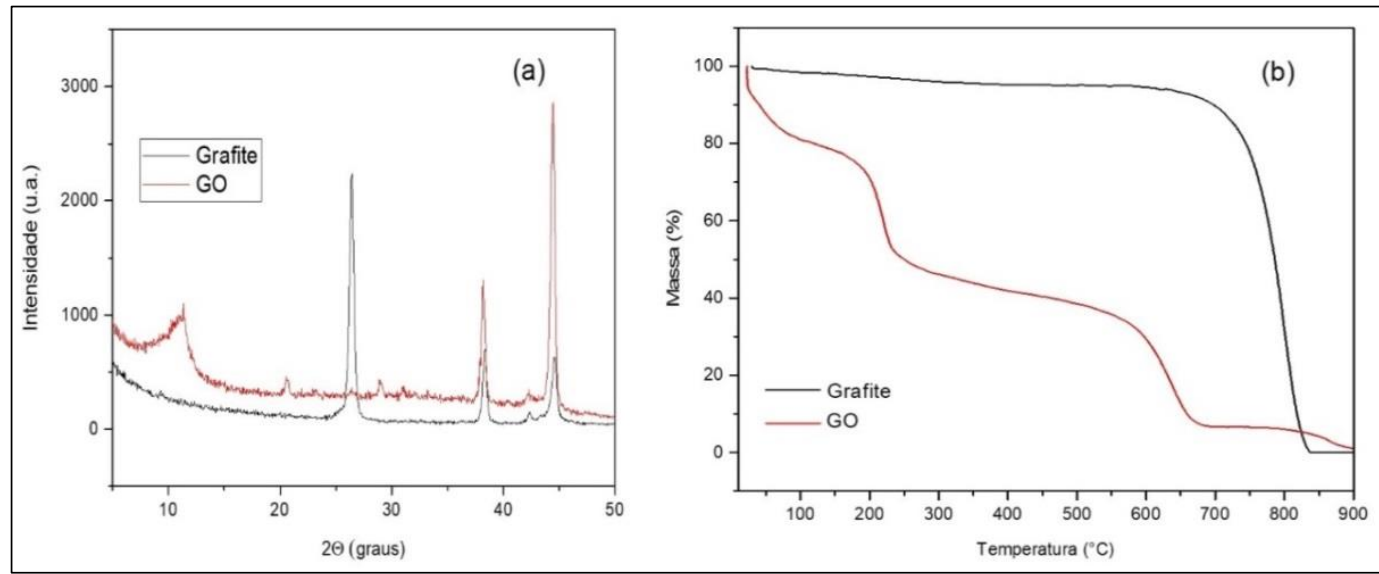

Figura 2: (a) Difratogramas de raios X e (b) curvas TGA para amostras de grafite e GO.

Pela técnica de FTIR (Figura 3) foi possível identificar a presença dos grupos funcionais que compõem o GO. O espectro de FTIR para o GO exibe inúmeras bandas indicando grupos contendo oxigênio que confirmaram a oxidação do EG. A banda de absorção a $3392 \mathrm{~cm}^{-1}$ foi atribuída à vibração de O-H do grupo $\mathrm{C}-\mathrm{OH}$ e da água. A banda em $1728 \mathrm{~cm}^{-1}$ à vibração de estiramento do grupo $\mathrm{C}=\mathrm{O}$, em $1622 \mathrm{~cm}^{-1}$ são registradas as vibrações de domínios grafíticos não oxidados C=C, C-C), em $1412 \mathrm{~cm}^{-1}$ e $1070 \mathrm{~cm}^{-1}$ são vibrações de alongamento $\mathrm{C}-\mathrm{O}$ e $\mathrm{C}-\mathrm{O}-\mathrm{C}$ respectivamente, relacionadas ao grupo epóxi. Todas essas bandas de absorção são bandas características de GO que confirmam a preparação de GO pelo método de Hummers modificado $[27,28]$.

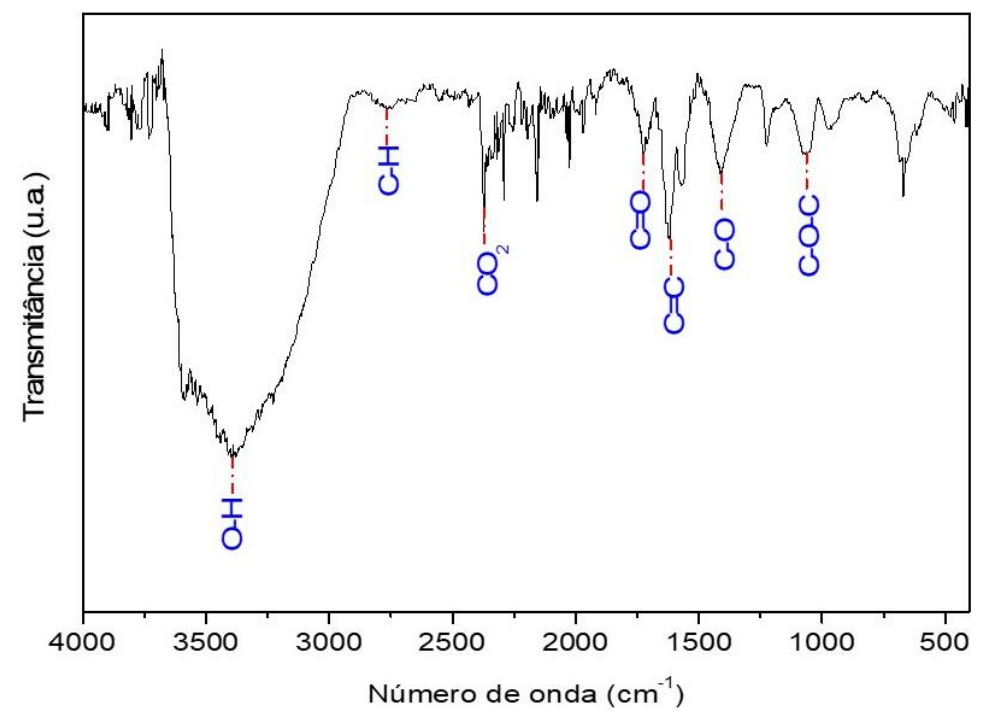

Figura 3: Espectro de FTIR para o GO.

\subsection{Estudo reológico dos nanofluidos de GO}

A Figura 4 mostra as curvas de viscosidades em relação a taxa de cisalhamento para os fluidos preparados. Estas curvas de fluxo confirmam a natureza pseudoplástica em baixos valores de taxa de cisalhamento. As moléculas estão em um estado desordenado, e quando submetidas a uma tensão de cisalhamento, estas tendem a se orientar na direção da força aplicada [31]. E quanto maior esta força, maior será a ordenação e, consequentemente, menor será a viscosidade aparente. Este fluido pode ser descrito pelo modelo de Ostwald-de Waele (1923) [32]. Com a adição de GO ao ETG é nítido o aumento da viscosidade do fluido base. O nanofluido ETG/GO-4 com uma concentração de $8 \mathrm{mg}_{\mathrm{mL}} \mathrm{mL}^{-1}$ possui um ganho percentual de $21 \%$ na viscosidade em relação ao ETG, comprovando o aprimoramento das propriedades deste com a adição de GO. De acordo com LIMA et al. [4], ao se utilizar nanoestruturas ocorre a viscosificação do meio, que é atribuída à formação de ligação de hidrogênio entre o GO e ETG, ocorrendo também o aumento na estabilidade à altas taxas de cisalhamento. 


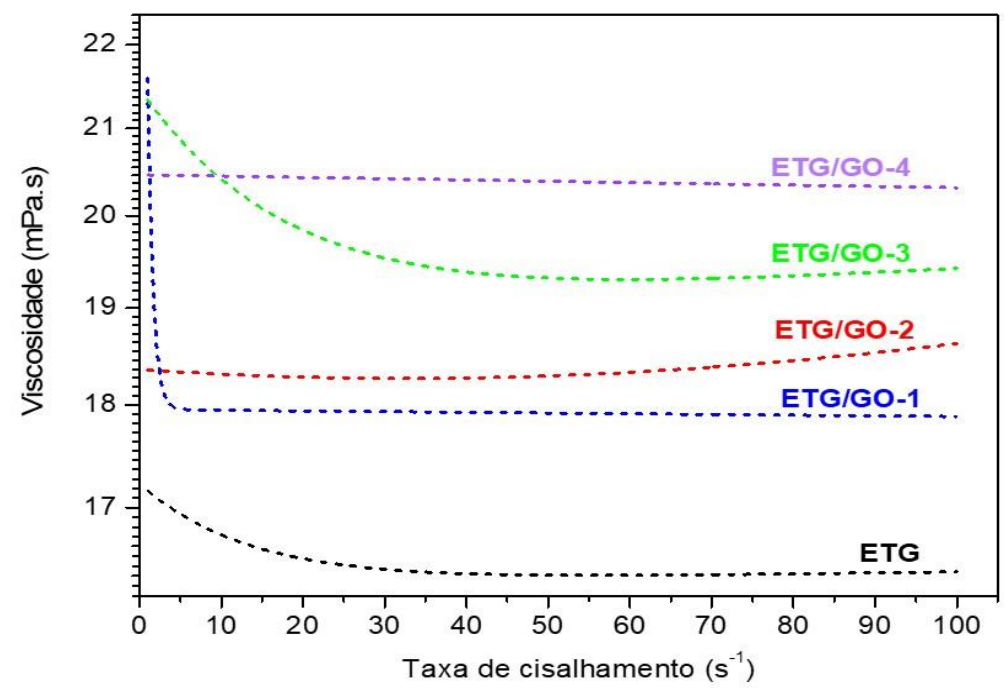

Figura 4: Curva de viscosidade para ETG e nanofluidos de ETG/GO.

Os próximos ensaios reológicos que serão apresentados, retratarão resultados do ETG e os nanofluidos ETG/GO-2 e ETG/GO-4 que, devido a diferença de concentração ser mais significativa, assinalaram um comportamento diferenciado comparado ao ETG. Foram feitos ensaios a fim de entender a correlação entre as concentrações e as propriedades reológicas destes nanofluidos.

No ensaio de varredura dinâmica de frequência, a frequência é variada, enquanto a amplitude de deformação permanece constante. De acordo com resultados obtidos apresentados na Figura 5, ao longo da faixa de frequência analisada, o módulo de armazenamento (G') tende a aumentar e superar o módulo de perda (G'), o que é característica intrínseca de dispersões estáveis [5, 10, 16]. As interações entre as folhas de GO causam respostas elásticas mais fortes do que as respostas viscosas na dispersão de GO, e este comportamento é observado durante uma grande faixa do ensaio, classificando o nanofluido de ETG/GO como uma dispersão estável. Isso se deve ao fato de intensas interações dos grupos funcionais do GO com o meio fluido em que se dispersa [25, 27]. Há um destaque para o nanofluido ETG/GO-4, que apresentou o módulo G' superior comparado ao G' em frequências menores que 0,12 rad.s ${ }^{-1}$. Um comportamento similar é observado para o ETG também. Apesar de haver uma transição de comportamento, em frequências acima de 6,3 rad.s $\mathrm{s}^{-1}$, o módulo de G' do nanofluido torna a ser superior ao módulo G'. Esse comportamento não é observado para ETG. O módulo G' está relacionada a energia mecânica que o material é capaz de armazenar, em determinadas condições, na forma de energia potencial ou elástica, e o módulo G' está relacionado a dissipação de energia [33]. Sendo assim, a adição de GO promove um aumento na capacidade de armazenamento de energia.

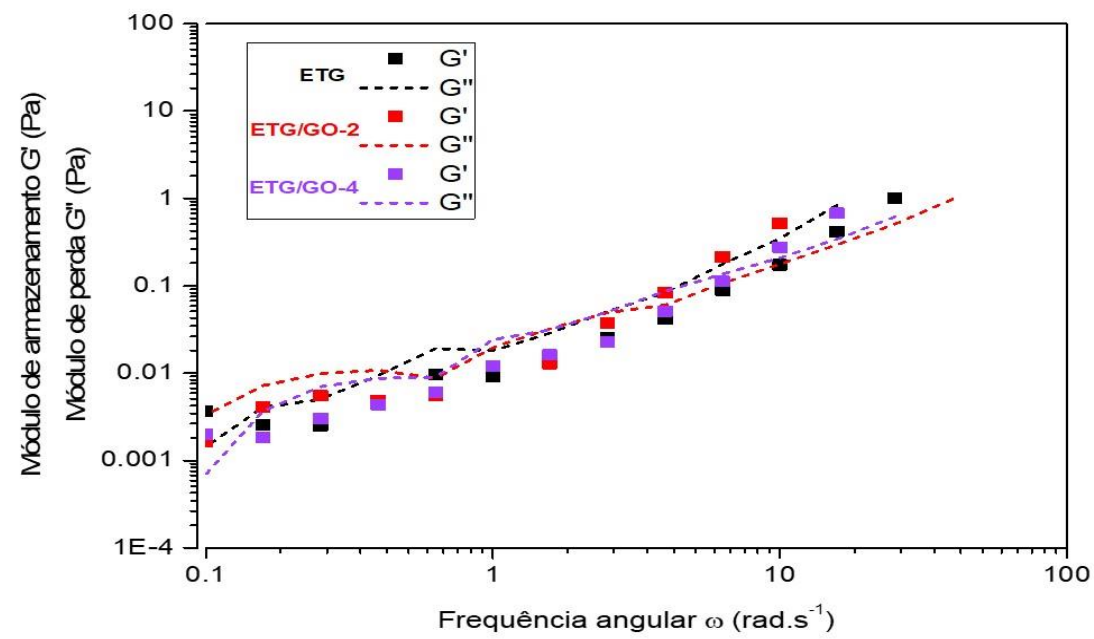

Figura 5: Curvas de módulos G' e G', em função da frequência angular para os fluidos preparados. 
No ensaio de varredura dinâmica de amplitude, a amplitude de deformação é variada, enquanto a frequência permanece constante em $1 \mathrm{~Hz}$. Na Figura 6, temos os resultados do ensaio de varredura de amplitude. Em baixas deformações, os módulos G' e G' se mantiveram constantes, os nanofluidos em estudo possuem uma região linear viscoelástica, ou seja, possuem uma resposta composta de uma parte elástica e outra viscosa, e as deformações são de pequena amplitude. As tensões resultantes oscilarão com a mesma frequência $\omega$, mas não estarão em fase com a deformação, exibindo então, um comportamento viscoelástico linear [5, 17, 24]. O módulo de perda (G’') se manteve superior ao longo do ensaio durante a deformação aplicada, ou seja, o módulo de perda é superior ao módulo de armazenagem (G’), pois, a energia de deformação é perdida pela fricção interna durante o cisalhamento, tendo as amostras comportamento de fluidos viscoelásticos [19, 24]. Na Figura 6, também é possível observar que os nanofluidos apresentaram comportamentos semelhantes, tendo a presença de uma região de viscoelasticidade linear (VEL). Estes também apresentaram pequena variação nos seus módulos G' e G' ' ao longo da taxa de deformação analisada, ou seja, os nanofluidos possuem independência da tensão de cisalhamento [19, 23]. Já o etilenoglicol puro, apresenta um comportamento diferente, pois, os módulos de G' e G', sofrem variações mais acentuadas.

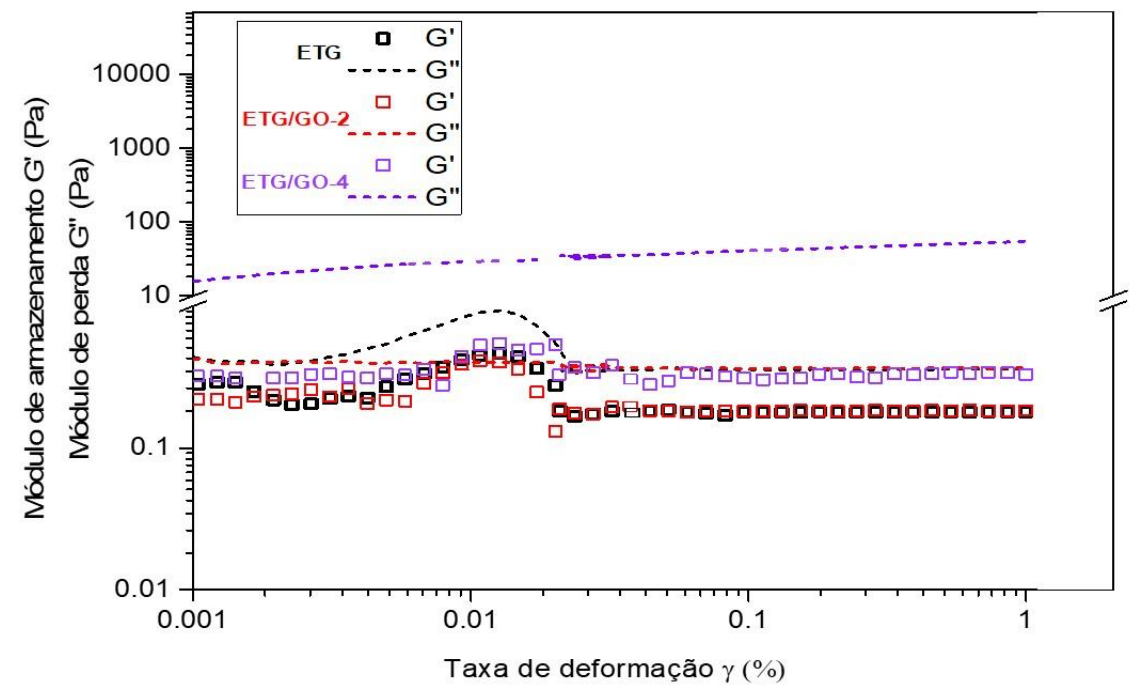

Figura 6: Curvas de módulos G’ e G’’ em função da deformação para os fluidos preparados.

Foi feita uma análise do comportamento das amostras em relação à temperatura em uma frequência constante de $1 \mathrm{~Hz}$. Utilizando os módulos G' e G', é possível ter informações sobre o reforço da matriz devido à presença de nanomateriais, como o GO. De acordo com SATAPATHY et al. [33], se o módulo G'> G', a amostra é mais sólida que líquida, ou seja, a amostra se torna mais viscosa e ocorre mudanças nas propriedades mecânicas. Na Figura 7, estão apresentados os resultados para o fluido base e os nanofluidos. O fluido ETG apresenta o módulo $G$ ' $>$ G' ao longo de toda faixa de temperatura analisada. Os nanofluidos possuem um comportamento diferente, uma vez que, com o aumento da temperatura o módulo G' > G', ou seja, com a adição de GO houve a viscosificação do fluido base devido à fortes interações entre o GO e ETG. Nota-se que, todas as amostras obtêm um comportamento estável em relação à temperatura, e que o ETG/GO-4 contém o G' superior desde o início do ensaio comparado ao ETG/GO-2, tendo um aumento de $8 \%$ no módulo de armazenamento devido a maior concentração de GO disperso. 

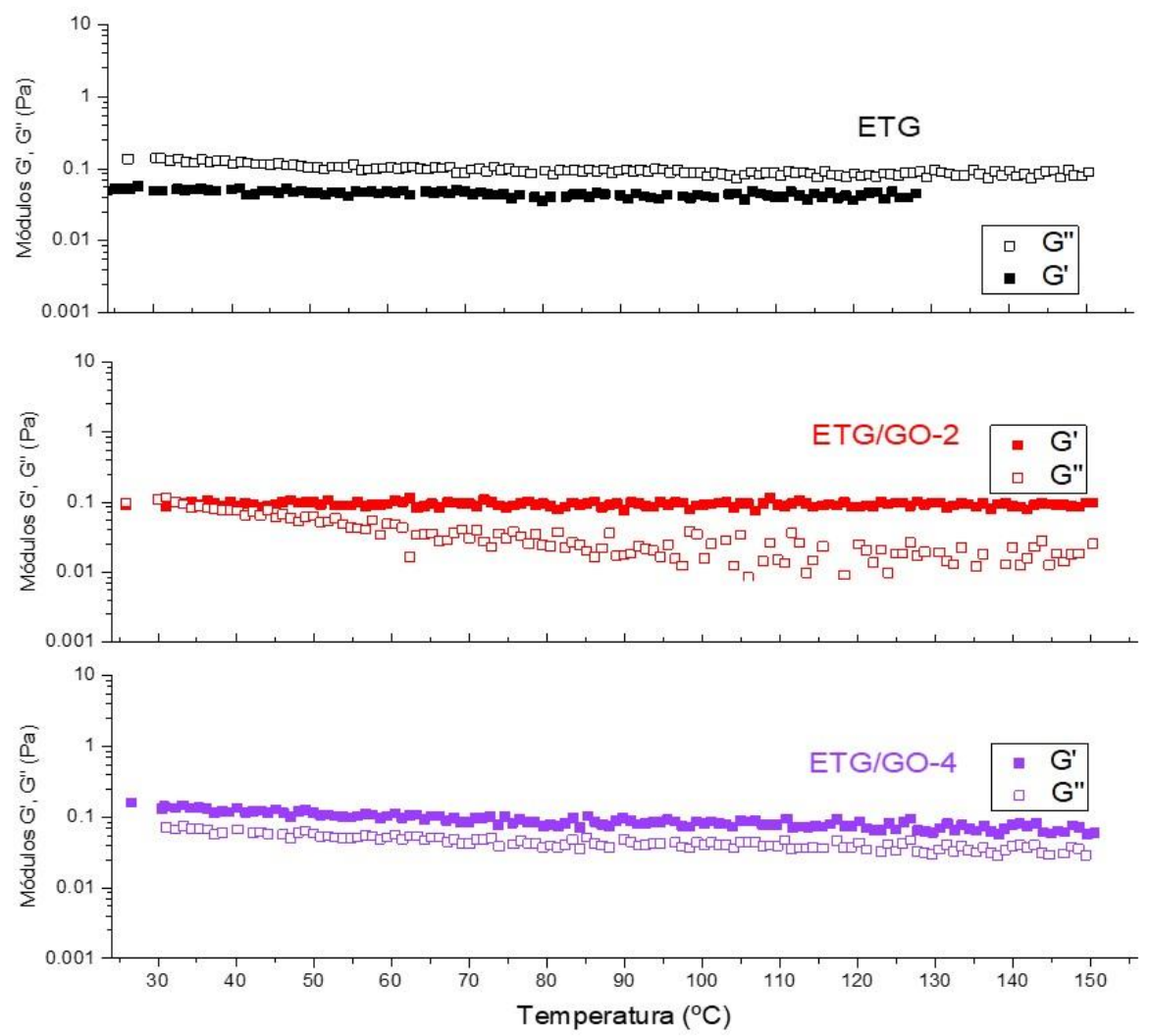

Figura 7: Curvas de módulos G' e G'” em função da temperatura para os fluidos preparados.

\section{CONCLUSÕES}

Nesse estudo, óxido de grafeno (GO) foi sintetizado pelo método Hummers modificado. Por meio das técnicas de DRX, MEV, MET, TGA e FTIR, comprovou-se a obtenção do GO com aproximadamente 4 folhas pelo método utilizado, ratificando a formação dos grupos funcionais oxigenados à superfície de grafeno, com um grau de funcionalização de $38 \%$. O estudo reológico foi feito através de quatro ensaios, envolvendo parâmetros de propriedades dinâmicas e mecânicas. A partir destes foram observados os seguintes comportamentos reológicos: (1) os nanofluidos obtiveram um comportamento não Newtoniano e pseudoplástico; sendo melhor ajustado pelo modelo Oswald-de Waele. Um percentual de viscosificação de até $21 \%$ devido ao acréscimo de GO em ETG foi observado; (2) obteve-se um aumento de $8 \%$ no módulo de armazenamento dos nanofluidos em relação ao ETG; (3) foi observada a presença da região de viscoelasticidade linear, na qual proporciona o comportamento duo dos nanofluidos, o que amplia o espectro de aplicação desses nanofluidos, cujas formulações podem ser planejadas e moduladas com a variação da concentração de GO para que determinado comportamento reológico seja alcançado; e (4) os nanofluidos apresentaram estabilidade durante o ensaio com varredura de temperatura, no qual o G' > G', ou seja, o comportamento viscoso é superior de modo que com a adição de GO em ETG, além de ocorrer a viscosificação do fluido base, existe também a estabilização da viscosidade em altas temperaturas, um fator que é propriedade fundamental de fluidos de troca térmica.

Os resultados encontrados para os nanofluidos ETG/GO desenvolvidos nesse trabalho podem ser aprimorados e testados em sistemas térmicos industriais, com o auxílio de softwares de modelagem e simulação, para o estudo de outros tipos de concentrações, regimes e fluxos, para aplicações específicas.

\section{AGRADECIMENTOS}

Agradecimentos aos órgãos de fomento à pesquisa que financiaram este projeto: Conselho Nacional de Desenvolvimento Científico e Tecnológico (CNPq), Coordenação de Aperfeiçoamento de Pessoal de Nível Superior - Brasil (CAPES) - Código de Financiamento 001 e Fundação de Amparo à Pesquisa do Estado de Minas Gerais (FAPEMIG). Ao laboratório de Microscopia pertencente ao IPUC-PUC Minas, pelas imagens e 
apoio técnico. Ao Dr. Getúlio Manoel pelo auxílio nas medidas reológicas. Ao grupo de pesquisa Gaiah. Ao Centro de Microscopia da Universidade Federal de Minas Gerais - CM / UFMG pelas análises de microscopia eletrônica (http://www.microscopia.ufmg.br).

\section{BIBLIOGRAFIA}

[1] NIKKAM, N., TOPRAK, M.S., DUTTA, J., et al., "Fabrication and thermo-physical properties characterization of ethylene glycol-MoS2 heat exchange fluids", International Communications in Heat and Mass Transfer, v. 89, pp. 185-189, Dez. 2017.

[2] HUMINIC, G., HUMINIC, A., "Hybrid nanofluids for heat transfer applications - A state-of-the-art review", International Journal of Heat and Mass Transfer, v. 125, pp. 82-103, Out. 2018.

[3] GUPTA, M., SINGH, V., KUMAR, R., et al., "A review on thermophysical properties of nanofluids and heat transfer applications", Renewable and Sustainable Energy Reviews, v. 74, pp. 638-670, Jul. 2017.

[4] LIMA, M.C.F.S., Fluidos de carbono nanoestruturados modificados com aminas e acrilamida, Tese de D. Sc., ICEx/UFMG, Belo Horizonte, MG, Brasil, 2015.

[5] NAFICY, S., JALILI, R., ABOUTALEBI, S.H., et al., "Graphene oxide dispersions: tuning rheology to enable fabrication", Materials Horizons, v. 1, n. 3, pp. 326- 331, Agos. 2014.

[6] ASADI, A., ASADI, M., REZANIAKOLAEI, A., et al., "Heat transfer efficiency of Al2O3-

MWCNT/thermal oil hybrid nanofluid as a cooling fluid in thermal and energy management applications: An experimental and theoretical investigation", International Journal of Heat and Mass Transfer, v. 117, pp. 474-486, Fev. 2018.

[7] MUHAMMAD, M.J., MUHAMMAD, I.A., SIDIK, N.A.C., et al., "The use of nanofluids for enhancing the thermal performance of stationary solar collectors", A review, Renewable and Sustainable Energy Reviews, v. 63, pp. 226-236, Set. 2016.

[8] SHAO, X.F., MO, S.P., CHEN, Y., et al. "Solidification behavior of hybrid TiO2 nanofluids containing nanotubes and nanoplatelets for cold thermal energy storage", Applied Thermal Engineering, v. 117, pp. 427436, Mai. 2017.

[9] ASADI, M., ASADI, A., ABEROUMAND, S., "An experimental and theoretical investigation on the effects of adding hybrid nanoparticles on heat transfer efficiency and pumping power of an oil-based nanofluid as a coolant fluid", International Journal of Refrigeration, v. 89, pp. 83-92, Mai. 2018.

[10] TESFAI, W., SINGH, P., SHATILLA, Y., et al., "Rheology and microstructure of dilute graphene oxide suspension”, Journal of Nanoparticle Research, v. 15, n. 10, Set. 2013.

[11] MARCANO D.C., KOSYNKIN, D.V., BERLIN, J.M., et al., "Improved synthesis of graphene oxide", ACS Nano, v. 4, n. 8, pp. 4806-4814, Agos. 2010.

[12] VIANA, M.M., LIMA. M.C.F.S., FORSYTHE, J.C., et al., "Facile Graphene Oxide Preparation by Microwave-Assisted Acid Method", Journal of the Brazilian Chemical Society, vol. 26, n. 5, pp. 978-984, Mai. 2015.

[13] THAKUR, V.K., THAKUR, M.K., Chemical functionalization of carbon nanomaterials: Chemistry and applications, 1 ed., Boca Raton, CRC Press, 2015.

[14] GUDARZI, M.M., "Colloidal Stability of Graphene Oxide: Aggregation in Two Dimensions", Langmuir, v. 32, pp. 5058-5068, Mai. 2016.

[15] SADEGHINEZHAD, E., MEHRALI, M., SAIDUR, R., et al., "A comprehensive review on graphene nanofluids: recent research, development and applications", Energy Conversion and Management, v. 111, pp. 466-487, Mar. 2016.

[16] MACOSKO C.W., Rheology: Principles, measurements, and applications, New York, Wiley-VCH, 1994.

[17] ÇENGEL, Y.A., CIMBALA, J.M., Mecânica dos Fluidos: Fundamentos e aplicações, 3 ed., São Paulo, AMGH, 2014.

[18] STEFFE, J.F., Rheological methods in food process engineering, 2 ed., Michigan, Freeman Press, 1996.

[19] DEL GIUDICE, F., SHEN, A.Q., "Shear rheology of graphene oxide dispersions", Current Opinion in Chemical Engineering, v.16, pp. 23-30, 2017. 
[20] RANJBARZADEH, R., AKHGAR, A., MUSIVAND, S., et al. "Effects of graphene oxide-silicon oxide hybrid nanomaterials on rheological behavior of water at various time durations and temperatures: Synthesis, preparation and stability", Powder Technology, v. 335, pp.375-387, Jul. 2018.

[21] ZHANG, H., WANG, S., LIN, Y., et al. "Stability, thermal conductivity, and rheological properties of controlled reduced graphene oxide dispersed nanofluids", Applied Thermal Engineering, v. 119, pp.132-139, Jun. 2017.

[22] SHU, R., YIN, Q., XING, H., et al. "Colloidal and rheological behavior of aqueous graphene oxide dispersions in the presence of poly (ethylene glycol)", Colloids and Surfaces A: Physicochemical and Engineering Aspects, v. 488, pp.154-161, Jan. 2016.

[23] DEMIRKIR, Ç., HAKAN, E., "Rheological and thermal characterization of graphene-water nanofluids: Hysteresis phenomenon”, International Journal of Heat and Mass Transfer, v.149, Mar. 2020.

[24] KHAN, W.A., ALI, M., IRFAN, M., et al., "A rheological analysis of nanofluid subjected to melting heat transport characteristics", Applied Nanoscience, v.10, pp.3161-3170, Jun. 2019.

[25] CHOUHAN, A., MUNGSE, H.P., KHATRI, O.P., "Surface chemistry of graphene and graphene oxide: A versatile route for their dispersion and tribological applications", Advances in Colloid and Interface Science, v. 283, Jun. 2020.

[26] LI, X., LE, Z., CHEN, X., et al. "Graphene oxide enhanced amine-functionalized titanium metal organic framework for visible-light-driven photocatalytic oxidation of gaseous pollutants", Applied Catalysis B: Environmental, v. 236, pp. 501-508, Mai. 2018.

[27] GUERRERO-CONTRERAS, J., CABALLERO-BRIONES, F., "Graphene oxide powders with different oxidation degree, prepared by synthesis variations of the Hummers method", Materials Chemistry and Physics, v. 153, pp. 209-220, Mar. 2015.

[28] ARADHANA, R., MOHANTY, S., NAYAK, S.K., "Comparison of mechanical, electrical and thermal properties in graphene oxide and reduced graphene oxide filled epoxy nanocomposite adhesives", Polymer, v. 141, pp. 109-123, Mar. 2018.

[29] ALMEIDA, C.G., TRINDADE, T.N.S.; SILVA, M.V.S., et al., "Avaliação do papel do Óxido de Grafeno (GO) na geração fotocatalítica de hidrogênio em sistemas binários (GO-CdS) e ternários (Pt-GO-CdS)", Química Nova, v. 41, n. 7, pp.748-755, Jul. 2018.

[30] MARASCHIN, T.G., CORREA, R.S., RODRIGUES, L.F., et al., "Chitosan Nanocomposites with Graphene-Based Filler", Materials Research, v. 22, Feb. 2020.

[31] BRETAS, R.E.S., ÁVILA, M.A.D'., Reologia de polímeros fundidos, 2 ed., São Paulo, EduFSCar, 2010. [32] SCHRAMM, G., A practical approach to rheology and rheometry, 2 ed., Karlsruhe, Thermo Haake Rheology, 1998.

[33] SATAPATHY, M.K., CHIANG W.H., CHUANG E.Y., et al., "Microplasma-assisted hydrogel fabrication: A novel method for gelatin-graphene oxide nano composite hydrogel synthesis for biomedical application", PeerJ, Jun. 2017.

\section{ORCID}

Marielle Mara da Silva

Bruno Rocha Santos Lemos

Marcelo Machado Viana https://orcid.org/0000-0002-4030-5787

https://orcid.org/0000-0001-7221-3492

https://orcid.org/0000-0002-6682-2504 